\title{
Phylogenomics of a putatively convergent novelty: did hypertrophied lips evolve once or repeatedly in Lake Malawi cichlid fishes?
}

\author{
C. Darrin Hulsey ${ }^{1 *} \mathbb{D}$, Jimmy Zheng ${ }^{2}$, Roi Holzman ${ }^{3}$, Michael E. Alfaro ${ }^{2}$, Melisa Olave ${ }^{1}$ and Axel Meyer ${ }^{1}$
}

\begin{abstract}
Background: Phylogenies provide critical information about convergence during adaptive radiation. To test whether there have been multiple origins of a distinctive trophic phenotype in one of the most rapidly radiating groups known, we used ultra-conserved elements (UCEs) to examine the evolutionary affinities of Lake Malawi cichlids lineages exhibiting greatly hypertrophied lips.

Results: The hypertrophied lip cichlids Cheilochromis euchilus, Eclectochromis ornatus, Placidochromis "Mbenji fatlip", and Placidochromis milomo are all nested within the non-mbuna clade of Malawi cichlids based on both concatenated sequence and single nucleotide polymorphism (SNP) inferred phylogenies. Lichnochromis acuticeps that exhibits slightly hypertrophied lips also appears to have evolutionary affinities to this group. However, Chilotilapia rhoadesii that lacks hypertrophied lips was recovered as nested within the species Cheilochromis euchilus. Species tree reconstructions and analyses of introgression provided largely ambiguous patterns of Malawi cichlid evolution.

Conclusions: Contrary to mitochondrial DNA phylogenies, bifurcating trees based on our 1024 UCE loci supported close affinities of Lake Malawi lineages with hypertrophied lips. However, incomplete lineage sorting in Malawi tends to render these inferences more tenuous. Phylogenomic analyses will continue to provide powerful inferences about whether phenotypic novelties arose once or multiple times during adaptive radiation.
\end{abstract}

Keywords: Adaptive radiation, East African Rift Lakes, Fatlips, Phylogenomics

\section{Background}

Phylogenies are critical to testing convergence. Evolutionary trees can provide the framework for determining whether similar phenotypes have multiple origins or whether these traits have arisen only a single time [1-6]. Molecular phylogenies of East African cichlids provided some of the first examples of using DNA sequence data to establish the repeated evolution of similar phenotypes in radiations inhabiting different lakes [7-10]. Yet, clarifying whether cichlid lineages have evolved traits convergently within particular cichlid radiations that inhabit the same lake has remained problematic [11-16]. Additionally, the short periods ( $<2$ mya) over which cichlid groups like those inhabiting Lake Victoria and Lake Malawi have diversified have made reconstructing the phylogenies of

\footnotetext{
* Correspondence: darrin.hulsey@uni-konstanz.de

'Department of Biology, University of Konstanz, Konstanz, Germany

Full list of author information is available at the end of the article
}

these adaptively radiating groups exceptionally difficult [17-23]. However, phylogenomic analyses could allow us to determine whether traits such as the elongate bodies of piscivores, distinctive color patterns of fish inhabiting rocky reefs, or even bizarre phenotypes like greatly hypertrophied lips have arisen multiple times in radiations like the Lake Malawi cichlids.

Hypertrophied, or greatly enlarged, lips have arisen several times independently across fish diversity. Lineages possessing hypertrophied lips are present in the sailfin silverside fishes from the Malili lakes of Sulawesi [24] as well as in the barb species inhabiting Lake Tana of Ethiopia [25, 26]. But the best-known group that exhibits hypertrophied lips are the cichlid fishes (Fig. 1). Cichlids exhibiting greatly hypertrophied lips have independently arisen in the Nicaraguan crater lakes, large South American rivers, Lake Tanganyika, Lake Victoria, and Lake Malawi [10, 27-33]. This phenotype is often associated with feeding from rocky surfaces, and is likely

(c) The Author(s). 2018 Open Access This article is distributed under the terms of the Creative Commons Attribution 4.0 International License (http://creativecommons.org/licenses/by/4.0/), which permits unrestricted use, distribution, and 


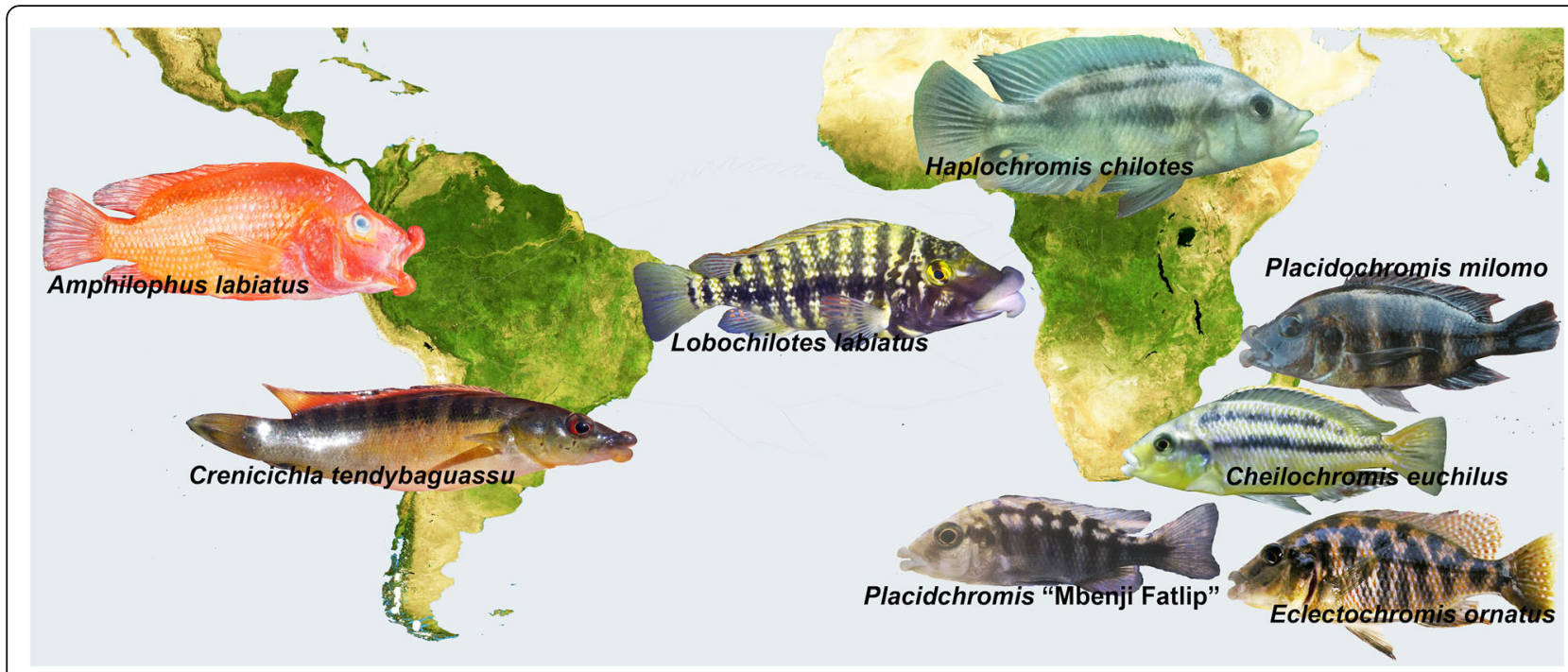

Fig. 1 Convergent evolution of hypertrophied lip phenotypes in cichlids fishes. This phenotype has arisen independently in a number of cichlid lineages inhabiting regions ranging from the Nicaraguan Rift Lakes (Amphilophus labiatus), South America (Crenicichla tendybaguassu), Lake Tanganyika (Lobochilotes labiatus), Lake Victoria (Haplochromis chilotes), and Lake Malawi (Cheilochromis euchilus, Eclectochromis ornatus, Placidochromis "Mbenji fatlip", and Placidochromis milomo). The lineages containing hypertrophied lips from these disparate geographic settings outside of Lake Malawi are well established as phylogenetically independent [79]. However, it is unclear if the hypertrophied lip phenotype has arisen convergently multiple times or alternatively only once within Lake Malawi

exceptionally effective for sucking prey from difficultto-access cracks and crevices [32, 34, 35]. The presence of hypertrophied lips could also form the basis of mate choice and ultimately speciation [36]. However, there is substantial plasticity in the size of the lips depending on the substrates individuals use during feeding, so the hypertrophied lip phenotype might be readily gained and lost during evolution [37].

The repeated evolution of hypertrophied lips across both a diversity of teleosts as well as its presence within numerous phylogenetically disparate cichlid lineages suggests that the hypertrophied lip lineages in Lake Malawi could have evolved convergently. With approximately 1000 species of haplochromine cichlids, the opportunity for the repeated origin of adaptive traits within the Malawi radiation is extensive [38-40]. Also, Malawi species with hypertrophied lips are currently classified into several different genera based in part on their extensive differences in body pigment patterns [27, 41-44]. For instance, the species Cheilochromis euchilus exhibits black horizontal stripes while Placidochromis milomo has vertical barred pigmentation that could indicate affinities with evolutionarily disparate Malawi clades (Fig. 1). Additionally, the Malawi hypertrophied lip species Placidochromis milomo has been inferred, based on mitochondrial DNA, to be phylogenetically nested within the rock-dwelling "mbuna" clade (Fig. 2) while other species with hypertrophied lips have been inferred to lie within the largely sand-dwelling, or non-mbuna, group of Malawi cichlids [22, 45-49]. However, phylogenies reconstructed using mitochondrial sequence markers have known limitations for phylogeny reconstruction $[17,18]$ and body pigmentation could often be a poor predictor of evolutionary affinities [31, 50].

Next-generation sequence based phylogenomic approaches have the potential to greatly clarify evolutionary patterns. For instance, sequence capture of large numbers of highly conserved regions of organismal genomes shared among evolutionary distant taxa, or ultra-conserved elements (UCEs), has been efficiently used to generate massive genomic data sets capable of resolving relationships at deep timeframes [51-56]. Additionally, one of the most compelling characteristics of UCEs for phylogenetic reconstruction is that the flanking regions increase in variant sites as the distance from the UCE center increases, thereby potentially allowing for resolution of nodes at more recent evolutionary timescales $[13,57,58]$. Yet, even with the ability to generate these massive datasets, the best approach to analyzing this type of sequence data in rapid radiations like Lake Malawi is largely unclear [13, 59, 60]. In Lake Malawi cichlids, single loci of from 500 to 1000 base pairs that are often used for phylogenetic reconstruction could frequently have only a few variable single nucleotide polymorphisms (SNPs) [23]. This lack of variation could make evolutionary inferences problematic. Furthermore, interspecific gene flow could be common in this largely sympatric radiation $[17,47,49,61]$ providing opportunities for extensive recombination during this group's divergence among even the few SNPs that do exist in a single sequence. Because of the vagaries of molecular evolution, 


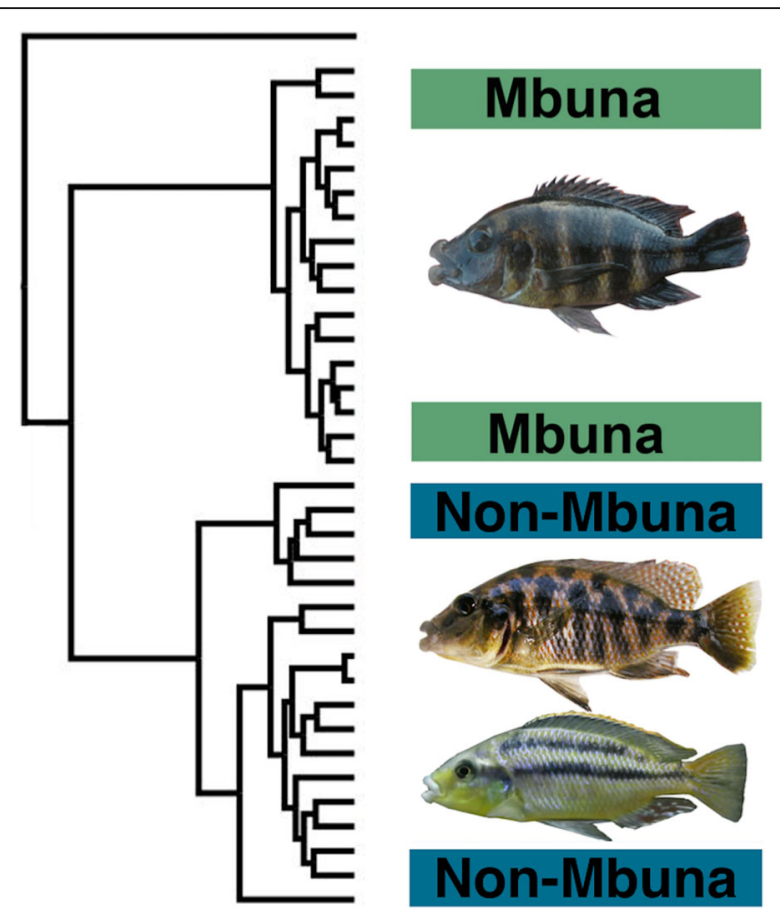

Fig. 2 Phylogenies of Malawi cichlids based largely on mitochondrial DNA have suggested that hypertrophied lip lineages are paraphyletic. This cartoon phylogenetic reconstruction of Malawi cichlids integrates the relationships inferred from numerous studies of Malawi phylogenetics that have primarily focused on mitochondrial genes [22, 45-49]. The hypertrophied lip species Placidochromis milomo has been inferred to be nested within the rock-dwelling mbuna radiation with high bootstrap support while the similarly hypertrophied lip species Placidochromis ornatus and Cheilochromis euchilus have been inferred to be nested within the largely sand-dwelling non-mbuna component of the Malawi radiation

model misspecification, as well as recombination, SNPs even in close proximity could often have unique evolutionary trajectories $[62,63]$. Therefore, in Lake Malawi cichlids, analyzing SNPs independently and as individual data points might provide tractable estimates of phylogeny.

However, it has become clear that incomplete sorting of loci among lineages has to be considered when reconstructing bifurcating phylogenetic relationships [64-67]. Malawi cichlids could provide a radiation that is exceptionally prone to gene tree discordance. This is not just because of the high level of incomplete lineage sorting expected in a recent radiation, but also because of the extensive sympatry of Malawi lineages that provides the opportunity for hybridization and the apparent lack of extensive post-zygotic incompatibilities [17, 23, 61, 68]. Therefore, methods that reconstruct the species tree while incorporating the potential for incomplete lineage sorting could provide an enhanced understanding of the Malawi phylogeny in general and the relationships among the hypertrophied lip species in particular. Furthermore, if hybridization was exceptionally rampant among hypertrophied lip species, we might view their reconstructed relationships differently than if hybridization was more common between hypertrophied lip species and other members of the Malawi cichlid radiation.

We used both sequence data and single nucleotide polymorphisms (SNPs) generated from genotyping a large number of UCE loci to infer the relationships among several lineages within Lake Malawi. First, we examined whether there was greater evidence for extensive paraphyly or alternatively monophyly of several lineages of Lake Malawi's hypertrophied lip species using concatenation methods that reconstruct a bifurcating topology. We also examined whether the UCE derived sequences and SNPs could provide insight into the relationships among the species with hypertrophied lips. Additionally, using sampling across geographically disparate sample sites in Lake Malawi for several congeneric species, we examined whether data from our UCE marker sets provided phylogenetic support for a number of taxonomically diagnosed clades using both concatenated analyses as well as species tree reconstructions that account for incomplete lineage sorting. Finally, we tested the support for hybridization among sampled members of the Malawi cichlid radiation and emphasized inferences of hybridization involving species with hypertrophied lips.

\section{Materials and methods}

All fishes sequenced in this study were collected from Lake Malawi in 2010 using SCUBA and barrier nets. Using a combination of 23 newly sequenced individuals and published sequences for 25 individuals, phylogenetic relationships among 35 species of Malawi cichlid were examined (Table 1). Our samples include five of the seven currently known Malawi species with hypertrophied lips. Multiple individuals of Placidochromis milomo, Cheilochromis euchilus, and Eclectochromis ornatus were sampled. Because of availability, only one individual of the hypertophied lip taxa Placidochromis "Mbenji fatlip" and Lichnochromis acuticeps were analyzed. Chilotilapia rhoadesii that is not a species that exhibits hypertrophied lips but is thought to be closely related to Cheilochromis euchilus was also examined [58]. Additionally, we included two species of the genera Labeotropheus, Pseudotropheus, Ctenophyarnx, Otopharynx, and Taeniolethrinops as well as three members of the genera Mylochromis, Placidochromis, and Nimbochromis. The East African species Pundamillia pundamillia, Haplochromis burtoni, and Simochromis babaulti were used as outgroups to polarize relationships. We reconstructed phylogenies using UCEs that show substantial conservation across teleosts and represent loci that have been sequenced previously for African cichlid fishes [13, 58]. Therefore, they should not show particular bias in their amplification or sequence divergence in the Malawi cichlids sampled. 
Table 1 Sampling of Specimens from Lake Malawi

\begin{tabular}{|c|c|c|c|}
\hline Species & Number of Loci & Sampling Location & Genbank Accession \\
\hline Astatotilapia burtoni & 1047 & commercial & [58] \\
\hline Aulonocara stuartgranti & 1042 & Maleri & [58] \\
\hline Cheilochromis euchilus & 1042 & Mbenji & [58] \\
\hline Cheilochromis euchilus & 1004 & Thumbi West & KARF00000000 \\
\hline Cheilochromis euchilus & 1007 & Mumbo & KAUH00000000 \\
\hline Chilotilapia rhoadesii & 1042 & commercial & [58] \\
\hline Ctenopharynx nitidus & 1012 & Choifu Point & KATA00000000 \\
\hline Ctenopharynx pictus & 1026 & Thumbi West & [13] \\
\hline Cyrtocara moorii & 1026 & Thumbi West & KARL00000000 \\
\hline Docimodus evelynae & 1024 & Boadzulu & [13] \\
\hline Eclectochromis ornatus & 1024 & Maleri & KATR00000000 \\
\hline Eclectochromis ornatus & 1024 & Maleri & KATS00000000 \\
\hline Eclectochromis ornatus & 1002 & Maleri & KAUJ00000000 \\
\hline Eclectochromis ornatus & 1020 & commercial & KAUI00000000 \\
\hline Fossorochromis rostratus & 1040 & Maleri & [58] \\
\hline Hemitilapia oxyrhyncha & 1018 & Mumbo & [13] \\
\hline Labeotropheus fuelleborni & 1036 & Thumbi West & [58] \\
\hline Labeotropheus trewavasae & 1036 & Thumbi West & [58] \\
\hline Lichnochromis acuticeps & 1016 & Thumbi West & KASA00000000 \\
\hline Mchenga conophoros & 1024 & Otter Point & [13] \\
\hline Melanochromis auratus & 1039 & Thumbi West & [58] \\
\hline Mylochromis anaphyrmus & 1009 & Harbor & KASU00000000 \\
\hline Mylochromis epichorialis & 1008 & Mbenji & KASV00000000 \\
\hline Mylochromis mola & 1008 & Otter Point & KASB00000000 \\
\hline Nimbochromis linni & 1021 & Thumbi West & KASW00000000 \\
\hline Nimbochromis livingstonii & 1017 & Chiofu Point & KASX00000000 \\
\hline Nimbochromis polystigma & 1042 & Otter Point & [58] \\
\hline Nyassochromis prostoma & 1003 & Mazinizi Reef & KASY00000000 \\
\hline Otopharynx heterodon & 1013 & Harbor & [13] \\
\hline Otopharynx lithobates & 1042 & Mumbo & [58] \\
\hline Placidochromis electra & 1037 & Chiofu Point & [58] \\
\hline Placidochromis milomo & 1049 & Chinyamwezi & [58] \\
\hline Placidochromis milomo & 1004 & Thumbi West & KATM00000000 \\
\hline Placidochromis milomo & 1013 & Thumbi West & KATN00000000 \\
\hline Placidochromis milomo & 1001 & Maleri & KATO00000000 \\
\hline Placidochromis milomo & 1019 & Nakatenga & KATP00000000 \\
\hline Pladichromis "Mbenji fatlip" & 1012 & Mbenji & KATQ00000000 \\
\hline Pseudotropheus crabro & 1046 & Maleri & [58] \\
\hline Pseudotropheus flavus & 1042 & Chinyankwezi & [58] \\
\hline Pundamilia pundamilia & 1047 & commercial & [58] \\
\hline Rhamphochromis longiceps & 1047 & commercial & [58] \\
\hline Simochromis babaulti & 1047 & commercial & [58] \\
\hline Stigmatochromis woodi & 1025 & Maleri & [13] \\
\hline Stigmatochromis woodi & 1018 & Chiofu Point & KATV00000000 \\
\hline
\end{tabular}


Table 1 Sampling of Specimens from Lake Malawi (Continued)

\begin{tabular}{llll}
\hline Species & Number of Loci & Sampling Location & Genbank Accession \\
\hline Taeniolethrinops furcicauda & 1009 & Chiofu Point & KATW000000000 \\
Taeniolethrinops praeorbitalis & 1011 & Otter Point & [13] \\
Taeniolethrinops praeorbitalis & 1024 & Otter Point & KATY00000000 \\
Tyrannochromis nigriventer & 1022 & Thumbi West & [13] \\
\hline
\end{tabular}

Species names, the number of loci sequenced, sampling location and Genbank Accession information are given for all individuals analyzed. All locations reported from this study are specific locations within Lake Malawi. Genbank accessions for a number of individuals are provided in McGee et al. [58] and Hulsey et al. (2017)

\section{DNA extraction and library preparation}

We extracted DNA from 5 to $15 \mathrm{mg}$ of ethanol-preserved tissues. We followed a modified version of the Qiagen DNEasy protocol, which utilizes $65 \mathrm{uL}$ of warm $\left(50-55^{\circ} \mathrm{C}\right)$ buffer $\mathrm{AE}$ instead of the recommended $200 \mathrm{uL}$ at room temperature. Following elution, we quantified extraction efficiency using a Qubit 2.0 Fluorometer by thoroughly mixing $2.0 \mathrm{uL}$ eluate with $198 \mathrm{uL}$ of fluorescent dye solution. To ensure high-quality extracts, we visualized 50-100 ng of each extract via electrophoresis using a $1.5 \%$ agarose gel in TBE. We then prepared $100 \mathrm{uL}$ aliquots for each specimen that were equilibrated to a DNA concentration of $10 \mathrm{ng} / \mathrm{uL}$ and then sonicated the aliquots using a BioRuptor (Diagenode, Inc.). Each sample was sheared to generate products of $300-500 \mathrm{bp}$ in length that were then size validated with gel visualizations.

Following sonication, we prepared libraries according to a modified version of the Illumina library preparation protocols from [52]. In preparing pooled DNA libraries, we used a series of standard library preparation reagents (Kapa Biosystems, Inc.) combined with dual-indexing adaptors [69] that we added during the PCR amplification phase. By doing so, we substantially reduced the number of primer tags needed to uniquely identify and differentiate libraries. Immediately after, we quantified the nucleic acid concentrations of pre-amplification libraries. Following quantification, we prepared a $50 \mathrm{uL}$ PCR reaction mix consisting of 15-20 uL DNA library, $25 \mathrm{uL}$ HiFi HotStart ReadyMix polymerase, $5 \mathrm{uL}$ primer mix, and $0-5 \mathrm{uL}$ double-distilled water $(\mathrm{ddH} 2 \mathrm{O})$. The following thermal cycle configuration was used: $98^{\circ} \mathrm{C}$ for $45 \mathrm{~s}, 10-16$ cycles of $98^{\circ} \mathrm{C}$ for $15 \mathrm{~s}, 60^{\circ} \mathrm{C}$ for $30 \mathrm{~s}, 72^{\circ} \mathrm{C}$ for 60 s, then $72^{\circ} \mathrm{C}$ for an extended $5 \mathrm{~min}$, and an indefinite hold at $4{ }^{\circ} \mathrm{C}$. As a final step, we purified resulting reactions with $1.8 \mathrm{X}$ Serapure solution [69], two 80\% EtOH washes, and rehydrated purified samples with $23 \mathrm{uL} 10-\mathrm{mM}$ Tris buffer.

\section{Library enrichment and sequencing}

To prepare libraries for enrichment, the libraries were combined into pools of equimolar ratios $(\sim 500 \mathrm{ng}$ per pool). To normalize the volumes of each pool, pools were dried down in a SpeedVac and rehydrated in 3.4 uL Tris buffer. Based on the sequence capture protocol available at ultraconserved.org, libraries were enriched for UCE targets using the following reagents: (1) $100 \mathrm{ng}$ of the MYBaits UCE Capture Kit baits (MYcroarray, Inc.) (2) 500 ng blocking oligos designed against our custom dual sequence indexes, (3) MYcroarray MySelect hybridization solutions (MYcroarray, Inc.), and (4) $1 \%$ SDS (versus $10 \%$ SDS). The hybridization reaction was run for $24 \mathrm{~h}$ at $65^{\circ} \mathrm{C}$, allowing the capture probes to bind to UCE targets. Upon completion, we thoroughly mixed streptavidin-coated beads (MyOne C1, Life Technologies, Inc.) with the hybridized pools and then washed the bound libraries according to the protocol. Beads were then rehydrated in $33 \mathrm{uL}$ of ddH2O, amplified with $15 \mathrm{uL}$ of the mix in a post-hybridization limited cycle PCR recovery step, and the end products quantified using a Qubit fluorometer [52]. After qPCR-quantification of the enriched, doubleindexed pools using a library quantification kit (Kapa Biosystems), we created an equimolar solution of all pools at a total concentration of $10 \mathrm{nM}$. These libraries were then shipped to the Georgia Genomics Facility and sequenced using the Illumina NextSeq PE150 platform.

\section{Sequence data assembly and alignment}

Following sequencing, we trimmed adapters, low quality bases, and sequences containing ambiguous base calls using the Illumiprocessor tool [70] that provides a wrapper for the trimmomatic package [71]. The reads were assembled on a species-by-species basis into contigs using Trinity v2013-02-25 [72]. Following assembly, the PHYLUCE software package [73] containing custom Python code that integrates LASTZ to align species-specific contigs to the set of UCE probes was used for enrichment $[53,58]$. This program creates a relational database of matches to UCE loci sorted by taxon. After generating the relational database of matches to enriched sequences and genome-enabled taxa, we used additional components of PHYLUCE to query the database and generate fasta files for the UCE loci we identified across all taxa [73]. Following enrichment and sequencing, contigs that either matched no UCEs or contigs that matched multiple loci were removed. Using the remaining set of contigs, a matrix was generated that included only UCE loci that were recovered from at least $95 \%$ of the species examined. The data are available on Genbank's short read archive database (Table 1). 


\section{Concatenated phylogeny reconstruction}

To reconstruct phylogenetic hypotheses from our data, we concatenated our UCE alignments (Additional file 1) into a PHYLIP-formatted super-matrix [74].

We first carried out phylogenetic reconstruction on a 95\% complete matrix with a GTR + gamma partitioning scheme using RAxML 8.0.19 [75] and the PTHREADS binary. Initially, 20 maximum-likelihood (ML) searches were conducted to find the best-fitting phylogenetic hypothesis. Then, we generated non-parametric bootstrap replicates under the autoMRE flag which runs the analysis until convergence. Upon completion, the best fit ML tree was reconciled with the bootstrap replicates to generate node support values.

To reconstruct phylogenetic trees using SNPs, we aligned all raw reads against the sample with the highest coverage across all UCE loci and utilized a de novo SNP calling approach as described in Hulsey et al. 2017 [13]. This method integrates BWA v. 0.7.7-1 and PICARD v. 1.106 (http:// broadinstitute.github.io/picard//) to output alignments in BAM format, repairs any formatting violations, adds read group header information, and marks duplicates in each BAM. We then merged all resulting BAMs into one file, realigned the data, and called SNPs using GATK v. 3.5. To ensure high-quality SNPs in downstream analyses, the data was hierarchically filtered according to stringent quality and validation parameters, excluding SNPs with quality scores under 25 , low variant confidence, and poor validation. Finally, the resulting data was filtered further using VCFTOOLS v. 0.1.14 [76] to remove all loci that missed SNP calls for over $25 \%$ of the species (Additional file 2).

We reconstructed SNP trees using two data sets. The first data set included all SNPs recovered with a minor allele frequency greater than $2 \%$. Because linkage disequilibrium of SNPs in the same locus could influence our phylogenetic results, the second data set was filtered to only include the highest quality SNP per locus, resulting in 1024 SNPs (Additional file 3). We then converted the SNP data format from genomic data structure to FASTA via R packages "gdsfmt" and "SNPRelate" [77]. Then, we created a Phylip interleaved alignment file (Additional file 4) and ran the file through the PHYLIP program DNAML to infer a maximum likelihood tree [77]. Subsequently, 1000 non-parametric bootstrap replicates of the maximum likelihood tree were generated using the bootstrap.pml function in the R package "phangorn" as implemented in the SNPhylo pipeline $[78,79]$. As a final step, the maximum likelihood phylogeny was reconciled with the bootstrap replicates to compute node support values.

\section{Species tree reconstruction}

Using our conservative data set of a single SNP per locus, we reconstructed a species tree that accounts for incomplete lineage sorting using the coalescent-based SVDquartets program [80], implemented in PAUP v4.163 [81]. We evaluated all possible quartet combinations to produce the species tree. All individuals sampled from a species were used in the coalescent model allowing these individuals to inform the tree reconstruction. To assess confidence in nodes recovered, we generated 100 bootstrap replicate quartet trees from the 1024 SNPs.

\section{Testing for hybridization}

We tested for the level of interspecific gene flow present in our sampling of Malawi cichlids and concentrated on inferences of gene flow involving the hypertrophied lip species. To do this, we used the program HyDe [82] to test for hybridization among all possible triplet combinations of species. HyDe uses phylogenetic invariants, similar to the D-statistic [83], to assess statistically significant evidence for hybridization. This was again implemented on our single SNP per locus data set of 1024 SNPs. Specifically, we employed the python script run_hyde.py to test all possible triplet comparisons among our sampled cichlids. Because all the possible triplet combinations for 37 taxa $\left(\frac{37}{3}\right) \times 3=23,310$ hypotheses tests, we assessed significance using a Bonferroni correction of $(0.05) /(23,310)=$ $2.15 \times 10^{-6}$.

\section{Results}

Following enrichment and sequencing, an average of 4,910,117 reads and 94,430 contigs were obtained per species. The analyzed matrix included only loci that were recovered from at least $95 \%$ of the species examined, constituting 1024 UCEs that had an average length of $324 \mathrm{bp}$. The assembled alignments included 563,696 base pairs of sequence. The alignments contained 10,465 total SNPs prior to filtering, 2707 when only SNPs with a minor allele frequency greater than $2 \%$ were included, and 1024 when only one SNP per locus for the 1024 UCE loci were included. We then generated phylogenies using RAxML on sequences (Additional file 5) as well as SNPhylo for the 2707 SNPs (Additional file 6), and for the 1024 SNPs (Additional file 7).

The RAxML inferred phylogeny for all the loci was generally less resolved than the SNP inferred phylogenies (Fig. 3). However, there was clear bootstrap support $(100 \%)$ for both a monophyletic mbuna clade and a large non-mbuna clade that included all of the species with hypertrophied lips in the RAxML tree. Also, species with multiple individuals sampled were often recovered as monophyletic. A few notably supported nodes (100\%) included those for the monophyly of the species sampled from the genera Nimbochromis, Ctenochromis, Taeniolethrinops, and Labeotropheus. Yet, many of the relationships among the non-mbuna, including those for the 


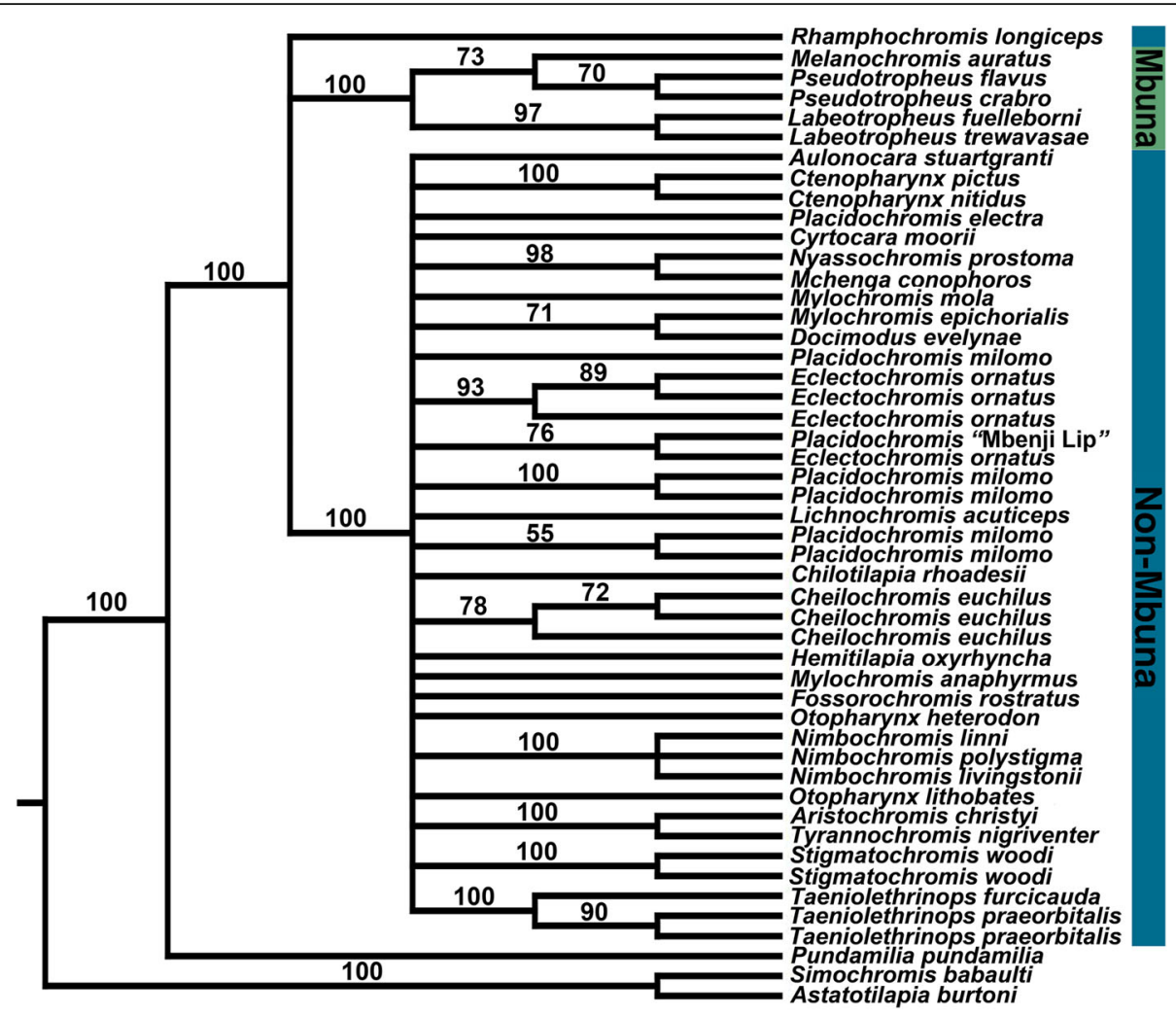

Fig. 3 RAxML phylogenetic reconstruction of Malawi cichlds using 1024 UCEs. The reconstruction was based on a matrix was that included only UCE loci that were recovered from at least $95 \%$ of the species examined. The consensus topology is shown and bootstrap values greater than $50 \%$ from the combined searches are given behind the nodes. In general, there was clear support (100\%) for the monophyly of both an mbuna clade and a large non-mbuna clade that included all of the species with hypertrophied lips. Many of the relationships among the non-mbuna, including those for the hypertrophied lip species, were not resolved here although species with multiple individuals sampled were often recovered as monophyletic

hypertrophied lip species, were not well-resolved $(<50 \%$ bootstraps) in this RAxML inferred phylogeny. Also, only the relatively poorly supported (71\%) relationship of Docimodus evelynae and Mylochromis epichoralis was not recovered in the SNP inferred phylogenies.

Our concatenated SNP tree reconstructions provided novel phylogenetic inferences of Malawi cichlid evolutionary relationships (Fig. 4). We recovered strong support for Rhamphochromis longiceps as sister to the remaining diversity of the Malawi cichlids examined. As in the RAxML tree and a previous study [13], we recovered the relatively unambiguous monophyly of two major clades that respectively contained the rock-dwelling mbuna sampled and a clade of primarily sand-dwelling nonmbuna cichlids. The cichlids with hypertrophied lips were all recovered as nested within this non-mbuna clade. In general, the data sets analyzed using both the 1024 loci with RAxML and 1024 SNPs were generally consistent but provided less support than the relationships inferred using the 2707 SNP data set which we report on in detail below.
The 2707 concatenated SNPs supported the monophyly of a clade containing the species Placidochromis milomo, Placidochromis "Mbenji fatlip", Eclectochromis ornatus, and Cheilochromis euchilus as well as the non-hypertrophied lip species Chilotilapia rhoadesii. Lichnochromis acuticeps grouped with these taxa greater than $50 \%$ of the time in the data set of 2707 SNPs. Within the hypertrophied lip group of species, Placidochromis "Mbenji fatlip" was recovered as sister to the monophyletic sampling of Eclectochromis ornatus (90\% bootstraps in the larger SNP dataset). The individuals of Placidochromis milomo were also recovered as monphyletic (100\%). In greater than $50 \%$ of the trees reconstructed using the 2707 SNPs, Placidochromis milomo was sister to the grouping of Cheilochromis euchilus + Chilotilapia rhoadessi.

In addition to the hypertophied lip clade, a number of taxonomically diagnosed lineages were also recovered as monophyletic with close to $100 \%$ bootstrap support. The members of the genus Labeotropheus were found to be monophyletic (100\%). Additionally, both species of Taeniolethrinops were recovered as monophyletic (100\%) as 


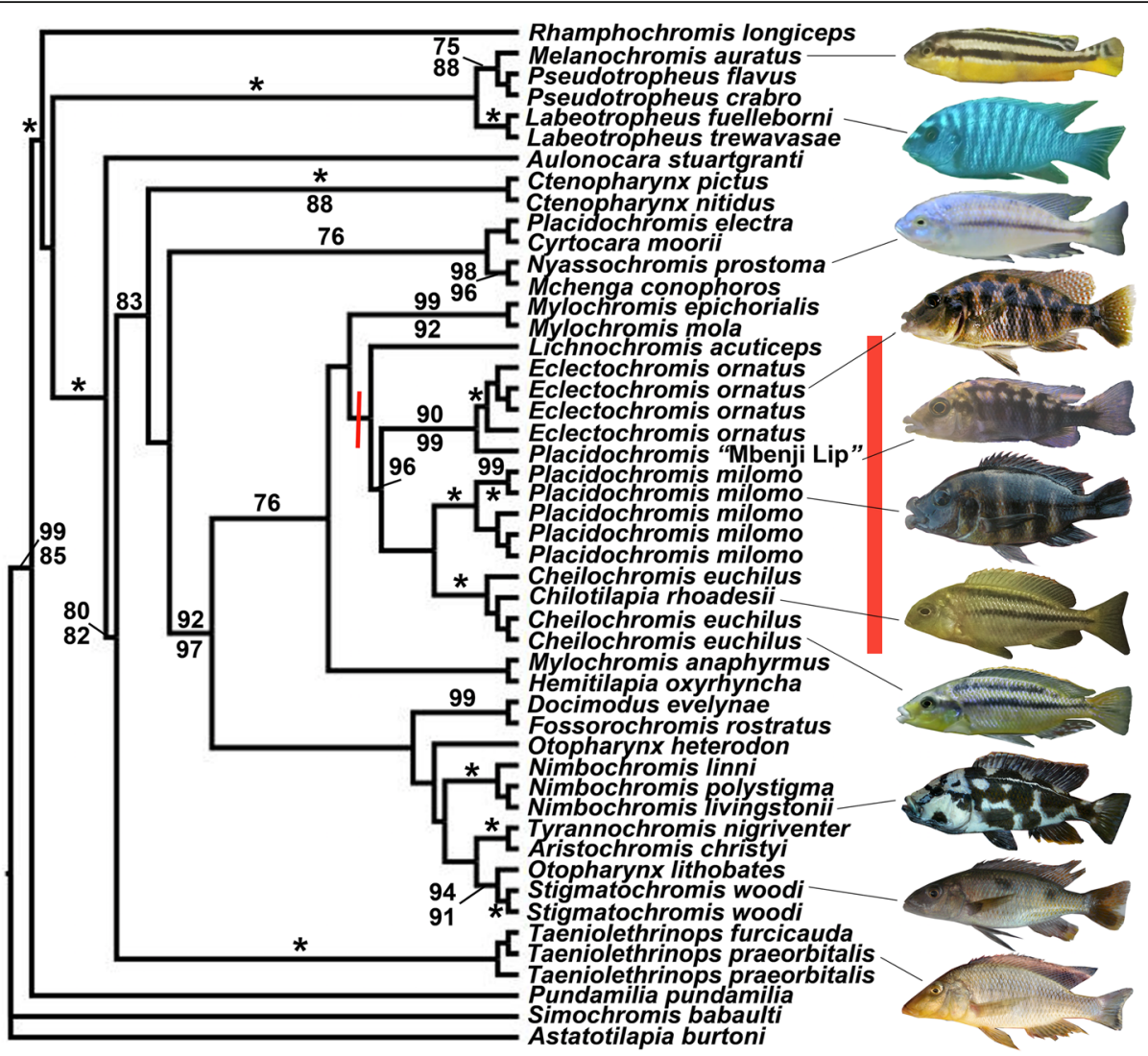

Fig. 4 Phylogenetic reconstruction of UCE generated SNPS. The consensus topology inferred using the 2707 SNPs that had a minor allele frequency greater than $2 \%$ is presented. The bootstrap values of the 2707 SNP dataset are shown above the branches subtending nodes, and bootstraps from the data set limited to a single SNP per locus are shown below the branches. There is evidence for the monophyly, shown with a bar dissecting the branch that subtends their last common ancestor, of the hypertrophied lip species Eclectochromis ornatus, Placidochromis "Mbenji fatlip", Placidochromis milomo, and Cheilochromis euchilus as well as the non-hypertrophied lip species Chilotilapia rhoadesii. This group is highlighted with a red bar behind the species names. Also, Lichnochromis acuticeps, which has somewhat hypertrophied lips, groups with these taxa greater than $50 \%$ of the time in the 2707 SNP dataset

were the two individuals of Stigmatochromis woodi (100\%) sequenced. Furthermore, the three species of Nimbochromis, $N$. linni, $N$. polystigma, and N. livingstonii formed a strongly supported monophyletic clade (100\%).

A number of other relationships recovered were noteworthy. Aulonocara stuartgrantii was recovered as the sister group to the remainder of the large clade of nonmbuna. Placidochromis was not a monophyletic genus, as P. electra had strong affinities to Cyrtocara moorii and did not group with the hypertrophied lip Placidochromis species. Mylochromis mola and M. epichorialis were recovered as monophyletic, but Mylochromis anaphyrmus had somewhat unclear affinities with these other Mylochromis. As previously documented [13], Tyrannochromis nigriventer and Aristochromis christyi were found to form a clade (100\%). Likewise, Docimodus evelynae and Fossorochromis rostratus were strongly supported (99\%) as closely related. The SNPs provided substantial resolution not only for taxonomically recognized groups but also for some clades that have not been proposed previously.
The SNP species tree reconstruction using SVDQuartets provided reduced resolution of relationships among lineages (Fig. 5). Following bootstrapping of SNPs, a limited number of clades were recovered in greater than $50 \%$ of the replicates. A sister group relationship of Nimbochromis livingstonii and N. polystigma were supported (56\%) and their inclusion in a clade with $N$. linni was better supported (76\%). Mchenga conophoros and Nyassochromis prostoma were recovered as having fairly strong phylogenetic affiinites (85\%) and were minorly supported (57\%) as aligned to a monophyletic group containing Taeniolethrinops furcicauda and $T$. praeorbitalis. Pladichromis "Mbenji Lip" and Eclectochromis ornatus was supported as a clade in 53\% of reconstructions while Cheilochromis euchilus and Chilotilapia rhoadesii were recovered as sister in $98 \%$ of reconstructions. However, Lichnochromis acuticeps was not recovered as part of a hypertrophied lip clade. Nevertheless, the remaining hypertrophied lip clade was recovered as monophyletic, but in general there was limited resampling support $(<50 \%)$ for 


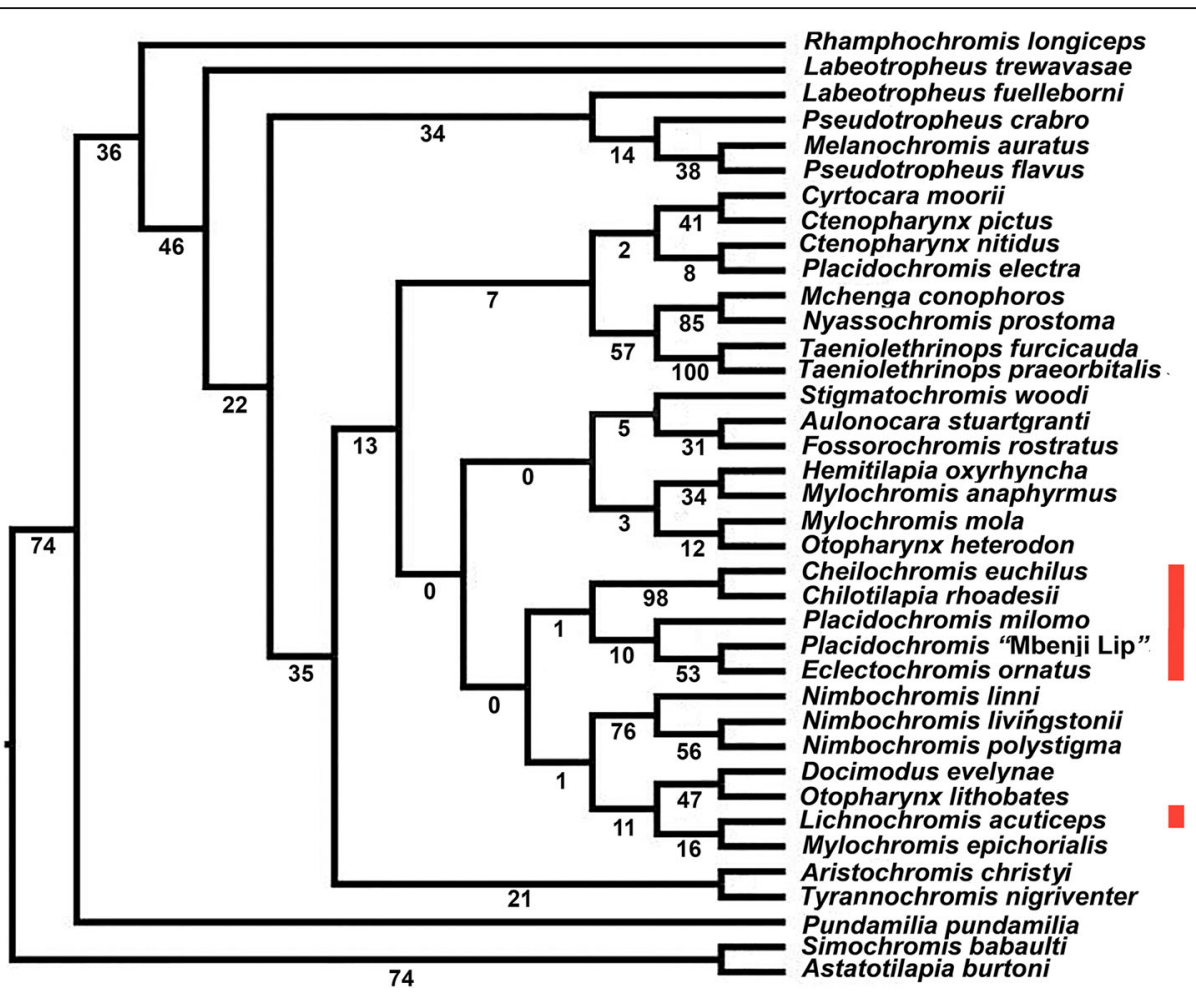

Fig. 5 Quartet inferred species tree. To account for incomplete lineage sorting, we reconstructed the species tree of the Malawi cichlids sampled using the one SNP per locus data matrix analyzed in SVDquartets. The phylogeny inferred using the complete data matrix is shown, and bootstrap replicates of the data were used to generate the support values shown behind each node recovered in the original data matrix tree. All individuals sampled from a species were used in the coalescent model. This allowed these individuals to inform the species tree reconstruction, but in the above diagram they are collapsed into their respective species. Few of the nodes exhibit substantial bootstrap support. The species exhibiting hypertrophied lips are again highlighted with a red bar to the right of the species names

the existence of a monophyletic hypertrophied lip species tree clade.

Following the Bonferroni correction for multiple comparisons (23,310 comparisons) among all possible triplets (Additional file 8), none of the tests for introgression remained significant. Nevertheless, since introgression in the Malawi cichlids seems to be a potential factor in inferring evolutionary histories, we examined the top 50 most significant cases for introgression further. When we examined these 50 triplets, 12 of the top 50 inferences involved hypertrophied lip species (Table 2). However, only two of these involved triplets containing more than one hypertrophied lip species. Interestingly, in both cases the marginally hypertrophied lip species, Lichnochromis acuticeps, was inferred to be involved. However, in general there was no overwhelming evidence that the other hypertrophied lip species were exhibiting substantial introgression with one another.

\section{Discussion}

The lineages in Lake Malawi that have hypertrophied lips based on concatenated sequence analyses appear to all fall within a relatively closely related and largely monophyletic
Table 2 HyDe inferences of Malawi cichlid hybridization

\begin{tabular}{llll}
\hline P1 & Hybrid & P2 & P-value \\
\hline P. flavus & O. heterodon & P. “Mbenji fatlip” & 0.0015 \\
L. trewavasae & H. oxyrhyncha & E. ornatus & 0.0022 \\
L. trewavasae & H. oxyrhyncha & P. “Mbenji fatlip” & 0.0023 \\
L. trewavasae & M. mola & E. ornatus & 0.0040 \\
P. crabro & L. acuticeps & P. “Mbenji fatlip” & 0.0114 \\
A. stuartgranti & M. conophoros & P. “Mbenji fatlip” & 0.0122 \\
T. praeorbitalis & M. conophoros & P. “Mbenji fatlip” & 0.0124 \\
P. “Mbenji fatlip" & E. ornatus & L. acuticeps & 0.0125 \\
L. trewavasae & P. electra & P. “Mbenji fatlip” & 0.0144 \\
C. pictus & C. nitidus & P. “Mbenji fatlip” & 0.0148 \\
P. electra & H. oxyrhyncha & C. rhoadesii & 0.0159 \\
R. longiceps & M. anaphyrmus & E. ornatus & 0.0174 \\
\hline
\end{tabular}

Inferences of hybridization [82] among Malawi cichlids and members of the 'hypertrophied lip' clade as shown in bold. We examined introgression occurring between triplets of three species: 1) An initial donor species (P1), 2) a hybrid species containing putatively introgressed loci, and 3 ) a second hybridizing donor 'hypertrophied lip' species (P2). Out of the 23,310 triplicates analyzed involving all the Malawi species sampled (Additional file 8), we depict only those inferred relationships involving a hypertrophied lip clade species that was recovered in the top 50 most likely hybridization events based on the $P$-values obtained from HyDe 
group (Figs. 3, 4). Contrary to the results from numerous studies with mitochondrial DNA [22, 45-49], all the species exhibiting hypertrophied lips that we sequenced are well-nested within the primarily sand-dwelling non-mbuna and none are nested within the mbuna. Our phylogenetic reconstructions employing more than 1000 loci from throughout the nuclear genome argue that there is only support for a single origin of the hypertrophied lip phenotype within the several hundred species that comprise the Lake Malawi cichlid radiation. Our results also lend credence to the idea that pigmentation is likely to be a questionable indicator of taxonomic affinities and phylogenetic relationships within Malawi cichlids [83]. Transformations from lateral to horizontal stripes characterize many lineages within East African cichlids [31, 50], and Malawi cichlids with hypertrophied lips could provide a model group to investigate the mechanisms involved in these changes in body patterning.

There remain several caveats to the apparent monophyly of the hypertrophied lip cichlid clade within Lake Malawi that we recovered. There are additional lineages of hypertrophied lips in Malawi that we did not sample $[41,44]$, and there could be additional lineages of cichlids with this lip phenotype remaining to be discovered in this radiation of up to 1000 species. For instance, a neighbor-joining reconstruction of whole genome sequences recently provided the inference that the species Placidochromis johnstoni and Hemitaeniochromis spilopterus, neither of which were sampled in this study and neither of which have hypertrophied lips, could be nested within the clade of hypertrophied lip Malawi cichlids [68]. Additionally, although Chilotilapia rhoadesii has long been thought to be allied taxonomically and phylogenetically with Cheilochromis euchilus, this nonhypertrophied lip species appears to be nested within the otherwise hypertrophied lip clade (Fig. 4). This suggests that the hypertrophied lip phenotype has likely been lost in this one species, but it could potentially indicate the repeated evolution of this phenotype in two closely related Malawi lineages. Furthermore, our phylogenetic reconstructions do enforce a strictly bifurcating topology and there is extensive retention of ancestral polymorphism and has likely been substantial introgression in the Malawi radiation $[17,47,49,61]$. Therefore, a bifurcating topology could provide misleading evidence of particular relationships [13]. However, despite these reservations, our analyses do provide evidence that the hypertrophied lip Malawi lineages are all apparently confined to the non-mbuna and show a closer affinity than has been previously appreciated (Fig. 2).

The SNPs generated from the sequencing of the 1000 UCE loci also provided substantial power when concatenated to support the monophyly of a number of previously diagnosed taxa (Fig. 4). Not only were congeneric species from several clades such as Otopharynx,
Nimbochromis, and Taeniolethrinops recovered as monophyletic, but this dataset provided the power to phylogenetically group multiple individuals sampled from the same species (Figs. 3,4). This level of resolution might seem trivial, but mitochondrial markers consistently support paraphyletic relationships of Malawi species [17, 61] and the RAxML tree provided poor resolution among most groups (Fig. 3). Interspecific gene flow could also commonly blur the genetic distinctiveness of species [47, 49]. However, these reconstructions argue that despite the substantial sequence polymorphism shared among different lineages of Malawi cichlids [17, 18, 20, 22, 23], some putatively bifurcating evolutionary relationships could be recoverable with sufficient information from the nuclear genome.

Although a few relationships were robustly recovered (> 85\% bootstrap support) in our species tree reconstructions (Fig. 5), our analyses incorporating incomplete lineage sorting provide little resolution of Malawi cichlid relationships. For instance, although the non-bootstrapped topology recovered a monophyletic hypertrophied lip clade minus Lichnochromis acuticeps, we only recovered this clade in a single bootstrap replicate. Additionally, based on our HyDe analyses (Table 2), there could be ample introgression among Malawi lineages, but none of them were significant after adjustment for the over twenty thousand comparisons made in the small subset of species sampled. Evaluating hybridization using phylogenies will only become more difficult if these hypotheses are evaluated when including additional species from this very species rich radiation. Furthermore, the lack of substantial evidence for introgression involving more than one member of the hypertrophied lip clade suggests hybridization is not an overt cause of the apparent close evolutionary affinities of these species. This all highlights that introgression in Malawi might be common [61, 68], but larger data sets that employ more sophisticated methods as well as clear a priori hypotheses of introgression will likely be necessary to reconstruct evolutionary relationships among the hundreds of Malawi cichlid species.

Because it is the most species-rich radiation of fishes in the world, the Lake Malawi cichlid radiation will continue to serve as a model of comparative phenotypic evolution [38]. However, many comparative analyses of these fishes have either discounted the importance of phylogeny when examining trait divergence or relied on the limited inferences of relationships available from mitochondrial gene trees $[46,48,84-86]$. With the advent of high throughput genotyping of markers such as UCEs, comparative analyses should be able to effectively leverage relatively robust phylogenetic hypotheses to make inferences concerning the number of times that particular traits have evolved within groups like the Lake Malawi cichlids [1-6]. For instance, our results using a large dataset of UCE loci support the hypothesis that 
hypertrophied lips might have only arisen once among the approximately 1000 species of Lake Malawi cichlids. Next generation sequence data will continue to shed new light on whether novel traits have evolved repeatedly or only a single time even in the most rapidly diversifying of adaptive radiations.

\section{Conclusions}

The Lake Malawi cichlid radiation provides an unparalleled model of comparative phenotypic evolution. Contrary to mitochondrial DNA phylogenies, bifurcating trees based on our 1024 UCE loci supported close affinities of Lake Malawi lineages with hypertrophied lips. Yet, future analyses will have to both collect more data and use more sophisticated analyses to account for incomplete lineage sorting. Phylogenomic analyses will continue to provide powerful inferences about whether phenotypic novelties arose once or multiple times during adaptive radiation.

\section{Additional files}

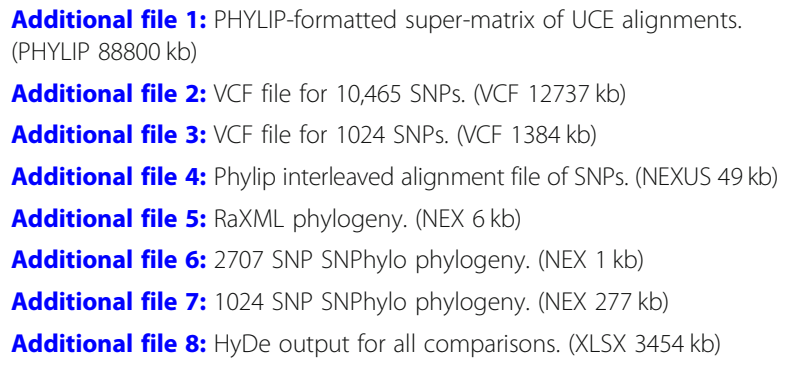

\section{Abbreviations}

BAM: Binary format for storing sequence data; DNA: Deoxyribonucleic acid; PCR: Polymerase chain reaction; SCUBA: Self-contained underwater breathing apparatus; SNP: Single nucleotide polymorphism; TBE: Tris-Borat-EDTA; UCE: Ultraconserved elements

\section{Acknowledgements}

We thank two anonymous reviewers and the Meyer lab group for helpful comments on the paper. Philip Hollingsworth also helped collect cichlids in Lake Malawi.

\section{Funding}

This work was partially funded through a grant from the U.S.-Israel Bi-National Science Foundation grant (BSF 2011067).

\section{Availability of data and materials}

The dataset supporting the conclusions of this article are available in the Genbank nucleotide repository with Genbank numbers available in Table 1. Sequence and SNP alignments, VCF files, and tree reconstructions are available as additional files associated with this publication. Phylogenies inferred using RAxML on sequences (Additional file 5) as well as SNPhylo for the 2707 SNPs (Additional file 6), and 1024 SNPs (Additional file 7) are also available as additional files

\section{Authors' contributions}

$\mathrm{CDH}, \mathrm{RH}$, and AM conceived of the study. JZ and MEA generated data for the study and performed several analyses. JZ performed the bioinformatic analyses and deposited the data on Genbank. MO performed a substantial number of analyses on the SNP species tree reconstructions and analyses of hybridization. $\mathrm{CDH}, \mathrm{MEA}, \mathrm{RH}, \mathrm{MO}$, and $\mathrm{AM}$ wrote the paper. All authors read and approved the final manuscript.

\section{Ethics approval}

All field work was approved by permits from the Malawi Parks Service in May 2010 to CDH Permit\#1 as well as the University of Tennessee-Knoxville Animal Care and Use Committee (IUCAC). We complied with all relevant guidelines for field collections of teleost fishes.

\section{Consent for publication}

All authors and relevant institutions have consented to this publication.

Competing interests

The authors declare that they have no competing interests.

\section{Publisher's Note}

Springer Nature remains neutral with regard to jurisdictional claims in published maps and institutional affiliations.

\section{Author details}

${ }^{1}$ Department of Biology, University of Konstanz, Konstanz, Germany. 2Department of Ecology \& Evolutionary Biology, University of California, Los Angeles, CA, USA. ${ }^{3}$ Department of Zoology, Tel Aviv University and the Inter-University Institute for Marine Sciences in Eilat, 88103 Eilat, Israel.

Received: 23 November 2017 Accepted: 16 November 2018 Published online: 29 November 2018

\section{References}

1. Wake DB. Homoplasy - the result of natural selection or evidence of design limitations. Am Nat. 1991;138:543-67.

2. Wake $\mathrm{DB}$, Wake $\mathrm{MH}$, Specht CD. Homoplasy: from detecting pattern to determining process and mechanism of evolution. Science. 2011;331:1032-5.

3. Bravo GA, Remsen JV, Brumfield RT. Adaptive processes drive ecomorphological convergent evolution in antwrens (Thamnophilidae). Evolution. 2014;68:2757-74.

4. Coghill LM, Hulsey CD, Chaves-Campos J, García de Leon FJ, Johnson SG. Next generation phylogeography of cave and surface Astyanax mexicanus. Molec Phylogen Evol. 2014;79:368-74.

5. Blom MPK, Horner P, Moritz C. Convergence across a continent: adaptive diversification in a recent radiation of Australian lizards. Proc R Soc B. 2016; 283:20160181.

6. Moen DS, Morlon H, Wiens JJ. Testing convergence versus history: convergence dominates phenotypic evolution for over 150 million years in frogs. Syst Biol. 2016;65:146-60

7. Meyer A, Kocher TD, Basasibwaki P, Wilson AC. Monophyletic origin of Lake Victoria cichlid fishes suggested by mitochondrial DNA sequences. Nature. 1990;347:550-3.

8. Kocher TD, Conroy JA, McKaye KR, Stauffer JR. Similar morphologies of cichlid fish in lakes Tanganyika and Malawi are due to convergence. Molec Phylogenet Evol. 1993;2:158-65.

9. Kocher TD, Conroy JA, McKaye KR, Stauffer JR, Lockwood SF. Evolution of NADH dehydrogenase subunit 2 in east African cichlid fish. Molec Phylogenet Evol. 1995;4:420-32.

10. Stiassny MLJ, Meyer A. Cichlids of the Rift Lakes. Sci Am. 1999;280:64-9.

11. Salzburger W, Meyer A. The species flocks of east African cichlid fishes: recent advances in molecular phylogenetics and population genetics. Naturwissenschaften. 2004:91:277-90.

12. Salzburger W, Mack T, Verheyen E, Meyer A. Out of Tanganyika: genesis, explosive speciation, key-innovations and phylogeography of the haplochromine cichlid fishes. BMC Evol Biol. 2005;5:17.

13. Hulsey CD, Zheng J, Faircloth BC, Meyer A, Alfaro ME. Phylogenomic analysis of Lake Malawi cichlid fishes: further evidence that the three-stage model of diversification does not fit. Molec Phylogen Evol. 2017;114:44-8.

14. Elmer KR, Meyer A. Adaptation in the age of ecological genomics: insights from parallelism and convergence. T.R.E.E. 2011;26:298-306.

15. Hulsey CD, Roberts RJ, Loh YHE, Rupp MF, Streelman JT. Lake Malawi cichlid evolution along a benthic/limnetic axis. Ecol Evol. 2013a;3:2262-72.

16. Hulsey CD, Keck BP, Alamillo H, O'Meara BC. Mitochondrial genome primers for Lake Malawi cichlids. Molec Ecol Res. 2013b;13:347-53. 
17. Moran P, Kornfield I. Retention of an ancestral polymorphism in the mbuna species flock (Teleostei, Cichlidae) of Lake Malawi. Molec Biol Evol. 1993;10: 1024-9

18. Meyer A. Shortcomings of the cytochrome $b$ gene as a molecular marker. T. R.E.E. 1994;9:278-80.

19. Delvaux D. Age of Lake Malawi (Nyasa) and water level fluctuations. Mus R Afr Centr Tervuren Dept Geol Min Rapp Ann. 1995-1996:99-108.

20. Loh EYH, Katz LS, Mims MC, Kocher TD, Yi SV, Streelman JT. Comparative analysis reveals signatures of differentiation amid genomic polymorphism in Lake Malawi cichlids. Genom Biol. 2008;9:R113.

21. Koblmüller S, Schliewen UK, Duftner N, Sefc KM, Katongo C, Sturmbauer C. Age and spread of the haplochromine cichlid fishes in Africa. Molec Phylogenet Evol. 2008;49:153-69.

22. Hulsey CD, Mims MC, Parnell NF, Streelman JT. Comparative rates of lower jaw diversification in cichlid adaptive radiations. J Evol Biol. 2010;23:1456-67.

23. Brawand D, Wagner CE, Li Yl, Malinsky M, Keller I, Fan S, et al. The genomic substrate for adaptive radiation in African cichlid fish. Nature. 2014; 513(7518):375-81.

24. Herder F, Nolte AW, Pfaender J, Schwarzer J, Hadiaty R, Schliewen UK. Adaptive radiation and hybridization in Wallace's Dreamponds: evidence from sailfin silversides in the Malili Lakes of Sulawesi. Proc R Soc Lond B Biol Sci. 2006;273:2209-17.

25. Sibbing FA, Nagelkerke LAJ, Stet RJM, Osse JWM. Speciation of endemic Lake Tana barbs (Cyprinidae, Ethiopia) driven by trophic resource partitioning: a molecular and ecomorphological approach. Aqu Ecol. 1998;32:217-27.

26. de Graaf M, Dejen E, Osse JWM, Sibbing FA. Adaptive radiation of Lake Tana's (Ethiopia) Labeobarbus species flock (Pisces, Cyprinidae). Mar Fresh Res. 2008:59:391-407.

27. Fryer G, lles TD. The cichlid fishes of the Great Lakes of Africa. Edinburgh: Oliver and Boyd; 1972

28. Colombo M, Diepeveen ET, Muschick M, Santos ME, Indermaur A, Bioileau N, Barluenga M, Salzburger W. The ecological and genetic basis of convergent thick- lipped phenotypes in cichlid fishes. Molec Ecol. 2012;22:670-84.

29. Burress ED, Duarte A, Serra WS, Loueiro M, Gangloff MM, Siefferman L. Functional diversification within a predatory species flock. PLoS One. 2013;8:e80929.

30. Manousaki T, Hull PM, Kusche H, Machado-Schiaffino G, Franchini P, Harrod C, Elmer KR, Meyer A. Parsing parallel evolution: ecological divergence and differential gene expression in the adaptive radiations of thick-lipped Midas cichlid fishes from Nicaragua. Molec Ecol. 2013;22:650-69.

31. Henning F, Meyer A. The evolutionary genomics of cichlid fishes: explosive speciation and adaptation in the postgenomic era. Ann Rev Genom Human Genet. 2014;15:417-41.

32. Baumgarten L, Machado-Schiaffino G, Henning F, Meyer A. What big lips are good for: on the adaptive function of repeatedly evolved hypertrophied lips of cichlid fishes. Biol J Linn Soc. 2015:115:448-55.

33. Burress ED. Cichlid fishes as models of ecological diversification: patterns, mechanisms, and consequences. Hydrobiologia. 2015;748:7-27.

34. Ribbink AJ, Marsh BA, Marsh AC, Ribbink AC, Sharp BJ. A preliminary survey of the cichlid fishes of rocky habitats in Lake Malawi. South Afric J Zool. 1983;18:149-310

35. Henning F, Machado-Schiaffino G, Baumgarten L, Meyer A. Genetic dissection of adaptive form and function in rapidly-speciating cichlid fishes. Evolution. 2017:71:1297-312.

36. Machado-Schiaffino G, Kautt AF, Torres-Dowdall J, Baumgarten L, Henning F, Meyer A. Incipient speciation driven by hypertrophied lips in Midas cichlids fish? Molec Ecol. 2017;26:2348-62.

37. Machado-Schiaffino G, Henning F, Meyer A. Species-specific differences in adaptive phenotypic plasticity in an ecologically relevant trophic trait: hypertrophic lips in Midas cichlid fishes. Evolution. 2014;68:2086-91.

38. Danley PD, Kocher TD. Speciation in rapidly diverging systems: lessons from Lake Malawi. Molec Ecol. 2001;10:1075-86.

39. Genner MJ, Seehausen O, Cleary DFR, Knight ME, Michel E, Turner GF. How does the taxonomic status of allopatric populations influence species richness within African cichlid fish assemblages? J Biogeogr. 2004;31:93-102.

40. Genner MJ, Turner GF. The mbuna cichlids of Lake Malawi: a model for rapid speciation and adaptive radiation. Fish Fisher. 2005;6:1-34

41. Arnegard ME, Snoeks J, Schaefer SA. New three- spotted cichlid species with hypertrophied lips (Teleostei: Cichlidae) from the deep waters of Lake Malawi/Nyasa, Africa. Copeia. 2001:705-17.
42. Snoeks J. The cichlid diversity of Lake Malawi/Nyasa/Niassa: identification, distribution and taxonomy. El Paso, Texas: Cichlid press; 2004. p. 360.

43. Konings A. Malawi cichlids in their natural habitat. 4th ed. El Paso, Texas: Oliver and Boyd; 2007.

44. Oliver MK, Arnegard ME. A new genus for Melanochromis labrosus, a problematic Lake Malawi cichlid with hypertrophied lips (Teleostei: Cichlidae). Ich Expl Fresh. 2010;21:209-32.

45. Won YJ, Sivasundar A, Wang Y, Hey J. On the origin of Lake Malawi cichlid species: a population genetic analysis of divergence. Proc Natl Acad Sci U S A. 2005;102:6581-6.

46. Hulsey CD, Mims MC, Streelman JT. Do constructional constraints influence cichlid craniofacial diversification? Proc Roy Soc Ser B. 2007;274:1867-75.

47. Joyce DA, Lunt DH, Genner MJ, Turner GF, Bills R, Seehausen O. Repeated colonization and hybridization in Lake Malawi cichlids. Curr Biol. 2011;21: R108-9.

48. York RA, Patil C, Hulsey CD, Streelman JT, Fernald RD. Evolution of bower building in Lake Malawi cichlid fish: phylogeny, morphology, and behavior. Front Ecol Evol. 2015;3:18.

49. Holzman R, Hulsey CD. Mechanical transgressive segregation and the rapid origin of trophic novelty. Sci Rep. 2017;7:40306.

50. Seehausen O, Mayhew PJ, Van Alphen JJM. Evolution of colour patterns in east African cichlid fish. J Evol Biol. 1999;12:514-34.

51. Bejerano G, Pheasant M, Makunin I, Stephen S, Kent WJ, Mattick JS, Haussler D. Ultraconserved elements in the human genome. Science. 2004;304:1321-5.

52. Faircloth BC, Branstetter MG, White ND, Brady SG. Target enrichment of ultraconserved elements from arthropods provides a genomic perspective on relationships among hymenoptera. Mol Ecol Res. 2014;15:489-501.

53. Faircloth BC, Sorenson L, Santini F, Alfaro ME. A phylogenomic perspective on the radiation of ray-finned fishes based upon targeted sequencing of ultraconserved elements (UCEs). PLoS One. 2013;8:e65923.

54. McCormack JE, Faircloth BC, Crawford NG, Gowaty PA, Brumfield RT, Glenn TC. Ultraconserved elements are novel phylogenomic markers that resolve placental mammal phylogeny when combined with species-tree analysis. Genome Res. 2012;22:746-54.

55. McCormack JE, Harvey MG, Faircloth BC, Crawford NG, Glenn TC, Brumfield RT. A phylogeny of birds based on over 1500 loci collected by target enrichment and high-throughput sequencing. PLoS One. 2013;8:e54848.

56. Smith BT, Harvey MG, Faircloth BC, Glenn TC, Brumfield RT. Target capture and massively parallel sequencing of ultraconserved elements for comparative studies at shallow evolutionary time scales. Syst Biol. 2014;63:83-95.

57. Faircloth BC, McCormack JE, Crawford NG, Harvey MG, Brumfield RT, Glenn TC. Ultraconserved elements anchor thousands of genetic markers spanning multiple evolutionary timescales. Syst Biol. 2012;61:717-26.

58. McGee MD, Faircloth BC, Borstein SR, Zheng J, Hulsey CD, Wainwright PC, Alfaro ME. Replicated divergence in cichlid radiations mirrors a major vertebrate innovation. Proc Roy Soc Ser B. 2016;283:20151413.

59. Bryant D, Bouckaert R, Felsenstein J, Rosenberg NA, Roy-Choudhury A. Inferring species trees directly from biallelic genetic markers: bypassing gene trees in a full coalescent analysis. Mol Biol Evol. 2012;29:1917-32.

60. Springer MS, Gatesy J. The gene tree delusion. Molec Phylogenet Evol. 2016;94:1-33.

61. Mims MC, Hulsey CD, Fitzpatrick BM, Streelman JT. Geography disentangles introgression from ancestral polymorphism in Lake Malawi cichlids. Molec Ecol. 2010;19:940-51.

62. Lanier HC, Knowles LL. Is recombination a problem for species-tree analyses? Syst Biol. 2012;61:691-701.

63. Edwards SV, Xi Z, Janke A, Faircloth BC, McCormack JE, Glenn TC, Zhong B, Wu S, Moriarty E. Implementing and testing the multispecies coalescent model: a valuable paradigm for phylogenomics. Molec Phylogenet Evol. 2016:20:447-62.

64. Maddison WP, Knowles LL. Inferring phylogeny despite incomplete lineage sorting. Syst Biol. 2006:55:21-30.

65. Kubatko LS, Degnan JH. Inconsistency of phylogenetic estimates from concatenated data under coalescence. Syst Biol. 2007:56:17-24

66. Degnan JH, Rosenberg NA. Gene tree discordance, phylogenetic inference and the multispecies coalescent. Trends Ecol Evol. 2009;24:332-40.

67. Leaché $A D$, Harris RB, Rannala B, Yang $Z$. The influence of gene flow on species tree estimation: a simulation study. Syst Biol. 2013;63:17-30.

68. Malinsky M, Svardal H, Tyers AM et al. Whole genome sequences of Malawi cichlids reveal multiple radiations interconnected by gene flow. bioRxiv preprint, 2018; https://doi.org/10.1101/143859. 
69. Glenn TC, Nilsen R, Kieran TJ, Finger JW, Pierson TW, Bentley KE, et al. Adapterama I: universal stubs and primers for thousands of dual-indexed Illumina libraries (iTru \& iNext). Molec Ecol Res. 2017; in press.

70. Faircloth BC. illumiprocessor: a trimmomatic wrapper for parallel adapter and quality trimming. 2013; https://doi.org/10.6079/J9ILL.

71. Bolger AM, Lohse M, Usadel B. Trimmomatic: a flexible trimmer for Illumina sequence data. Bioinformatics. 2014. https://doi.org/10.1093/bioinformatics/ btu170.

72. Grabherr MG, Haas BJ, Yassour M, Levin JZ, Thompson DA, Amit I, Adiconis $X$, et al. Full-length transcriptome assembly from RNA-Seq data without a reference genome. Nat Biotech. 2011;29:644-52.

73. Faircloth BC. PHYLUCE is a software package for the analysis of conserved genomic loci. Bioinformatics. 2016;32:786-8.

74. Felsenstein J. PHYLIP (Phylogeny Inference Package) version 3.6; 2005.

75. Stamatakis A. RAxML version 8: a tool for phylogenetic analysis and postanalysis of large phylogenies. Bioinformatics. 2014;30:1312-3.

76. Danecek P, Auton A, Abecasis G, Albers CA, Banks E, DePristo MA, et al. The variant call format and VCFtools. Bioinformatics. 2011;27:2156-8.

77. Zheng X, Levine D, Shen J, Gogarten S, Laurie C, Weir B. A highperformance computing toolset for relatedness and principal component analysis of SNP data. Bioinformatics. 2012;28:3326-8.

78. Schliep KP. Phangorn: phylogenetic analysis in R. Bioinformatics. 2011;27: 592-3.

79. Lee TH, Guo H, Wang X, Kim C, Paterson AH. SNPhylo: a pipeline to construct a phylogenetic tree from huge SNP data. BMC Genomics. 2014;15(1).

80. Chifman J, Kubatko L. Quartet inference from SNP data under the coalescent model. Bioinformatics. 2014;30:3317-24.

81. Swofford DL. Paup*: Phylogenetic analysis using parsimony (and other methods) 2001;4.0. B5.

82. Blischak PD, Chifman J, Wolfe AD, Kubatko LS. HyDe: a Python Package for Genome-Scale Hybridization Detection. Syst Biol. 2018;67:821-9.

83. Kidd MR, Kidd CE, Kocher TD. Axes of differentiation in the bower-building cichlids of Lake Malawi. Molec Ecol. 2006:15:459-78.

84. Meyer A. Phylogenetic relationships and evolutionary processes in east African cichlids. TREE. 1993;8:279-84.

85. Fraser GJ, Hulsey CD, Bloomquist RF, Uyesugi K, Manley NR, Streelman JT. An ancient gene network is co-opted for teeth on old and new jaws. PLoS Biol. 2009;7:233-47.

86. Friedman M, Keck BP, Dornburg A, Eytan Rl, Martin CH, Hulsey CD, Wainwright PC, Near TJ. Molecular and fossil evidence place the origin of cichlid fishes long after Gondwanan rifting. Proc Roy Soc Ser B. 2013;280: 20131733.

Ready to submit your research? Choose BMC and benefit from:

- fast, convenient online submission

- thorough peer review by experienced researchers in your field

- rapid publication on acceptance

- support for research data, including large and complex data types

- gold Open Access which fosters wider collaboration and increased citations

- maximum visibility for your research: over $100 \mathrm{M}$ website views per year

At $\mathrm{BMC}$, research is always in progress.

Learn more biomedcentral.com/submissions 